\title{
Reported fried food consumption and the incidence of hypertension in a Mediterranean cohort: the SUN (Seguimiento Universidad de Navarra) project
}

\author{
Carmen Sayon-Orea ${ }^{1}$, Maira Bes-Rastrollo ${ }^{1,2}$, Alfredo Gea ${ }^{1}$, Itziar Zazpe ${ }^{1,3}$, \\ Francisco J. Basterra-Gortari ${ }^{1,4}$ and Miguel A. Martinez-Gonzalez ${ }^{1,2 *}$ \\ ${ }^{1}$ Department of Preventive Medicine and Public Health, University of Navarra, Pamplona, Spain \\ ${ }^{2}$ CIBERobn Physiopathology of Obesity and Nutrition, Institute of Health Carlos III (ISCIII), Madrid, Spain \\ ${ }^{3}$ Department of Food Sciences, Physiology and Toxicology, University of Navarra, Pamplona, Spain \\ ${ }^{4}$ Department of Internal Medicine (Endocrinology), Hospital Reina Sofia, Tudela, Spain
}

(Submitted 10 January 2014 - Final revision received 7 May 2014 - Accepted 4 June 2014 - First published online 24 July 2014)

\begin{abstract}
Reported associations between the consumption of fried foods and the incidence of obesity or weight gain make it likely that fried food consumption might also be associated with the development of hypertension. However, evidence from long-term prospective studies is scarce. Therefore, the aim of the present study was to longitudinally evaluate this association in a prospective cohort. The SUN (Seguimiento Universidad de Navarra) project is a Mediterranean cohort study of university graduates conducted in Spain, which started in December 1999 and is still ongoing. In the present study, we included 13679 participants (5059 men and 8620 women), free of hypertension at baseline with a mean age of 36.5 (SD 10.8) years. Total fried food consumption was estimated at baseline. The outcome was the incidence of a medical diagnosis of self-reported hypertension during the follow-up period. To assess the association between the consumption of fried foods and the subsequent risk of developing incident hypertension during the follow-up period, Cox regression models were used. During a median follow-up period of 6.3 years, 1232 incident cases of hypertension were identified. After adjusting for potential confounders, the adjusted hazard ratios for developing hypertension were $1 \cdot 18$ (95\% CI $1 \cdot 03$, $1 \cdot 36$ ) and $1 \cdot 21$ (95\% CI 1.04 , 1.41) for those consuming fried foods $2-4$ and $>4$ times/week, respectively, compared with those consuming fried foods $<2$ times/week ( $P$ for trend=0.009). In conclusion, frequent consumption of fried foods at baseline was found to be associated with a higher risk of hypertension during the follow-up period in a Mediterranean cohort of university graduates.
\end{abstract}

Key words: Hypertension: Fried foods: Cohort studies

Hypertension is a strong and modifiable determinant for the development of several chronic diseases ${ }^{(1)}$. Hypertension is the first leading global risk factor for mortality in the world. It is responsible for $13 \%$ of deaths ${ }^{(2)}$ and 92 million disabilityadjusted life-years ( $6 \%$ of the total). Therefore, complications associated with hypertension account for $9 \cdot 4$ million deaths worldwide yearly ${ }^{(3)}$, and it is responsible for at least $45 \%$ of deaths due to heart disease and $51 \%$ of deaths due to stroke $^{(4)}$.

Available evidence supports the important role of food habits in the aetiology of hypertension ${ }^{(5)}$. Cooking methods may modify the role of some foods or nutrients with respect to the risk of hypertension. Among cooking methods, frying is one of the most common methods in Western countries. The consumption of fried foods is increasing due to their desirable characteristic flavour, brown colour and crispy texture, which make them very popular with consumers ${ }^{(6)}$. The frying process involves many factors: some factors are dependent on the process itself, whilst others are related to the type of food and fat that are used for frying ${ }^{(7)}$. According to previous studies such as the Seguimiento Universidad de Navarra (SUN) cohort study ${ }^{(8)}$ and the Pizarra study ${ }^{(9)}$, most people in Spain use olive oil for frying at home. In contrast, the most common oil used for frying away from home, especially in fast-food restaurants, is maize oil ${ }^{(10)}$.

Frequent fried food consumption has been reported to be associated with a higher risk of overweight/obesity in Spain ${ }^{(8,11)}$. This association together with some biological mechanisms (trans-fatty acids produced during the frying process may cause an impaired endothelial function, which will increase blood pressure) ${ }^{(12)}$ make it likely that fried food consumption might also be associated with a higher

Abbreviations: HR, hazard ratio; SUN, Seguimiento Universidad de Navarra.

* Corresponding author: Professor M. A. Martinez-Gonzalez, fax +34948 455649, email mamartinez@unav.es 
risk of hypertension. Evidence from epidemiological studies linking fried food consumption and hypertension is scarce and inconsistent. A cross-sectional study conducted in Spain has found that a high prevalence of hypertension is associated with the consumption of fried foods (especially when these foods are fried with reused vegetable oils such as sunflower oil) ${ }^{(13)}$. More evidence exists on the association between the consumption of fried foods and the risk of CVD. However, results of studies on the association between the consumption of fried foods and the risk of CVD are inconsistent: one case-control study ${ }^{(14)}$ and one cohort study $^{(15)}$ have found a positive association, while another cohort study ${ }^{(16)}$ and a case-control study ${ }^{(17)}$ have reported no association. It is widely known that unhealthy lifestyle factors such as overweight/obesity, physical inactivity, alcohol intake and smoking status, among others, cause high blood pressure $^{(18)}$. In one clinical trial, changes in unhealthy cooking habits (e.g. changing the cooking method from frying to boiling, among others) have been found to be beneficial for controlling blood pressure and obesity in the general population $^{(19)}$.

There are no published results from large prospective studies that have assessed the role of fried food consumption in the development of hypertension. Therefore, the aim of the present study was to prospectively evaluate the potential association between the consumption of fried foods and the risk of hypertension in a Mediterranean cohort.

\section{Subjects and methods}

\section{Study population}

The SUN project is a Spanish, multi-purpose, dynamic and prospective cohort study that was designed to assess associations between diet and the occurrence of several diseases and chronic conditions including hypertension ${ }^{(20)}$. All participants included in the study are university graduates. They are followed up every 2 years by mailed questionnaires. The recruitment of participants started in December 1999, and it is permanently open. Detailed information about the design and methods of the SUN project has been published elsewhere $^{(20)}$. Up to September 2009, 20335 participants had been recruited. We excluded 2283 participants with prevalent hypertension; 1747 participants who reported values for total energy intake at baseline outside the predefined limits $(<3347 \mathrm{~kJ} / \mathrm{d}(800 \mathrm{kcal} / \mathrm{d})$ in men and $<2092 \mathrm{~kJ} / \mathrm{d}$ $(500 \mathrm{kcal} / \mathrm{d})$ in women or $>16736 \mathrm{~kJ} / \mathrm{d}(4000 \mathrm{kcal} / \mathrm{d})$ in men and $>14644 \mathrm{~kJ} / \mathrm{d}(3500 \mathrm{kcal} / \mathrm{d})$ in women $)^{(21)}$; 1251 participants with chronic diseases at baseline (including cancer, diabetes or CVD); 1178 participants without any follow-up who were considered to be lost to follow-up (retention rate: 92\%); and 197 participants with missing values for variables of interest, leaving a total of 13679 participants available for the final analysis (Fig. 1).

The Institutional Review Board of the University of Navarra approved the study. Voluntary completion of the first selfadministered questionnaire was considered to indicate informed consent.

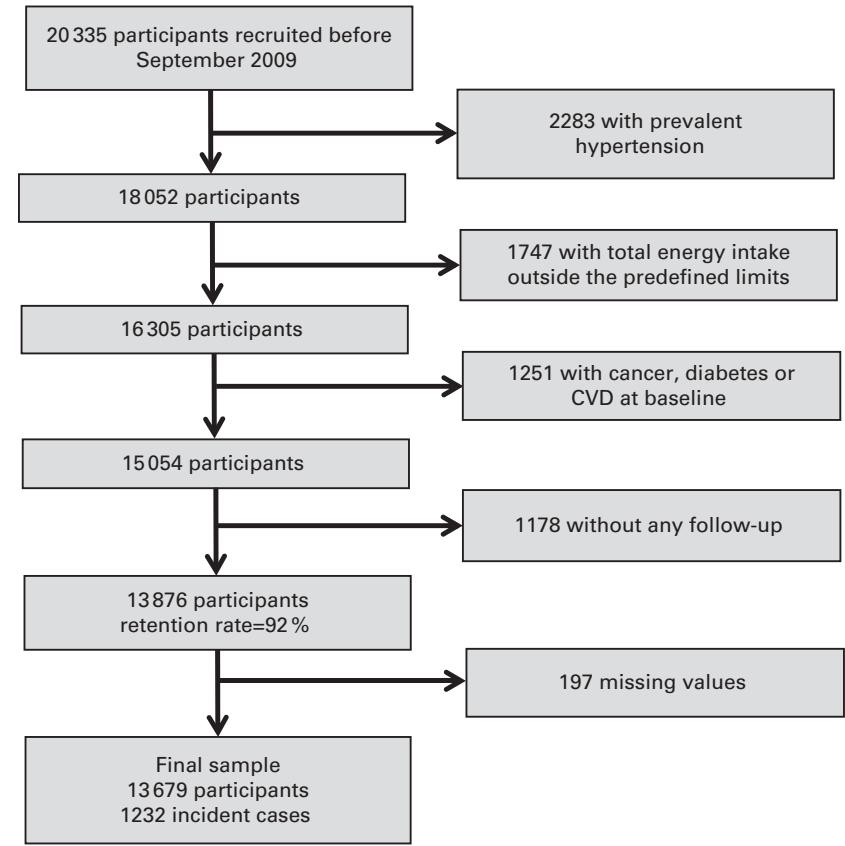

Fig. 1. Flowchart of participant recruitment: the SUN (Seguimiento Universidad de Navarra) project (1999-2012).

\section{Dietary intake assessment}

A previously validated 136 -item semi-quantitative FFQ measuring participants' food intake in the previous year was included in the baseline questionnaire ${ }^{(22)}$. Nutrient scores were calculated as frequency multiplied by the nutrient composition of specified portion sizes. For each food item, consumption frequencies were divided into nine categories (ranging from never/almost never, with almost never indicating that the specific item was consumed less than 'once per month', to $>6$ servings/d). The frequency of fried food consumption was evaluated by taking the following items into account: the consumption of fried foods at home and the consumption of fried foods away from home. The total consumption of fried foods per person was estimated using the sum of these two items. In addition, the total amount of fried foods consumed in grams was estimated by adding the proportion of fried foods consumed from the rest of the items in the FFQ. The proportion of consumption as a fried food according to typical Spanish culinary practices was multiplied by the frequency reported in the questionnaire (i.e. if the participant reported that he/she ate one portion of fried potatoes, the proportion of consumption was multiplied by one; on the other hand, if the participant reported that he/she ate one portion of meat, it was multiplied by 0.5 because we assumed that meat is consumed as a fried food half the time, taking into account the typical culinary practices in Spain). This approach has been used successfully in a previous study of the SUN cohort ${ }^{(8)}$. Finally, an additional question, 'what kind of fat do you use at home to fry?', was also included in the baseline questionnaire to determine whether the participants fried foods with olive oil or a different kind of oil. The nutrient databank was updated by a trained team of dietitians using the information available for food composition tables for Spain ${ }^{(23)}$. 


\section{Assessment of non-dietary variables}

Other questions (forty-six items for men and fifty-four items for women) were also included in the baseline questionnaire to assess participants' medical history, health-related habits, lifestyle, and sociodemographic variables, as well as anthropometric data (weight and height were validated previously in a subsample of the cohort ${ }^{(24)}$. At baseline, the participants were queried about seventeen activities to quantify their physical activity levels, and from them a metabolic equivalent index (MET-h/week) was computed. Physical activity had also been validated previously in a subsample of the cohort ${ }^{(25)}$.

\section{Ascertainment of hypertension}

The endpoint of the study was incident hypertension. The participants were asked whether they had received a medical diagnosis of hypertension at baseline and during the followup period and also about the date of diagnosis.

For the present analysis, the participants were considered to have prevalent hypertension at baseline if they reported a medical diagnosis of hypertension, systolic blood pressure $\geq 140 \mathrm{mmHg}$, diastolic blood pressure $\geq 90 \mathrm{mmHg}$, or use of any antihypertensive medication ${ }^{(26)}$. New cases of hypertension were defined as those participants who did not have hypertension at baseline and reported a new medical diagnosis of hypertension during the follow-up period. The validity of self-reported hypertension diagnosis was assessed in a subsample of the cohort. This validation study showed an adequate validity of the self-reported diagnosis of hypertension: among participants who reported a diagnosis of hypertension, $82 \cdot 3 \%$ (95\% CI 72.8, 92.8) were confirmed as hypertensive through conventional measurement of blood pressure, and among those who did not report a diagnosis of hypertension, $85 \cdot 4 \%$ (95\% CI $72 \cdot 4,89 \cdot 1)$ were confirmed as non-hypertensive ${ }^{(27)}$. Moreover, in a recent study, we have validated each component of the metabolic syndrome (including high blood pressure) and found an intra-class correlation coefficient of $0.47(0.36-0.57)$ for systolic blood pressure and of $0.46(0.34-0.56)$ for diastolic blood pressure, using direct assessments by an experienced physician as the gold standard $^{(28)}$.

\section{Statistical analyses}

The participants were classified into three categories according to the distribution of their frequencies of fried food consumption: $0-2 ;>2-4 ;>4$ times/week. The participants were also classified into tertiles of total fried food consumption $(\mathrm{g} / \mathrm{d})$ (details regarding food items and serving sizes of fried foods considered in the FFQ are given in an online supplemental table). The group with the lowest frequency of fried food consumption ( $<2$ times/week) or in the lowest tertile was considered as the reference category. In an additional analysis, the participants were classified into the following four categories: $0-2 ;>2-4 ;>4-<7 ; \geq 7$ times/week, considering the group with the lowest frequency of fried food consumption again as the reference category.
Nutrient intake was adjusted for total energy intake with the residuals methods, and separate regression models were used to obtain sex-specific residuals.

Person-time of follow-up was calculated for each participant, from the date of completion of the baseline questionnaire to the date of completion of the last follow-up questionnaire, the date of diagnosis of hypertension or death, whichever occurred first.

Cox regression models were used to assess the association between the consumption of fried foods at baseline and the subsequent risk of developing incident hypertension during the follow-up period. Tests of linear trend across increasing categories of fried food consumption were conducted by assigning medians for the frequency of fried food consumption within each category and treating this as a continuous variable.

The association between home and non-home fried food consumption separately and the risk of hypertension was also examined.

Interactions between age categories ( $<40$ and $\geq 40$ years), sex and the frequency of fried food consumption were calculated using likelihood ratio tests between the fully adjusted models and the same model, but by introducing the interaction product term. The interaction between the type of fat used for frying (olive oil or others) and the frequency of fried food consumption was also tested.

For all the analyses, a crude univariate model, an age- and sex-adjusted model, and two multivariable models were used after additional adjustment for the following potential confounders: model 1 - family history of hypertension (yes/no), self-reported history of hypercholesterolaemia (yes/no), physical activity (MET-h/week), smoking status (non-smoker, current smoker and former smoker), total energy intake $(\mathrm{kcal} / \mathrm{d})$, alcohol intake $(\mathrm{g} / \mathrm{d})$, and energyadjusted $\mathrm{Na}(\mathrm{mg} / \mathrm{d}), \mathrm{K}(\mathrm{mg} / \mathrm{d})$, caffeine $(\mathrm{mg} / \mathrm{d})$, fibre $(\mathrm{g} / \mathrm{d})$, olive oil $(\mathrm{g} / \mathrm{d})$, fruit $(\mathrm{g} / \mathrm{d})$, vegetable $(\mathrm{g} / \mathrm{d})$, low-fat and highfat dairy product, sugar-sweetened beverage $(\mathrm{ml} / \mathrm{d})$, fast food $(\mathrm{g} / \mathrm{d})$, and sweet $(\mathrm{g} / \mathrm{d})$ consumption, and time spent watching television $(\mathrm{h} / \mathrm{d})$ and model 2 - additionally adjusted for baseline BMI. For the selection of potential confounders in the multivariate model, and as currently recommended ${ }^{(29)}$, the previously published scientific literature, including our own results based on the cohort regarding risk factors for hypertension, was taken into account, avoiding exclusively the statistical approach with the $P$ value, the stepwise procedures or the changes in point estimates after adjusting for potential confounders.

To account for multicollinearity across independent variables, a statistical model was constructed and the variance inflation factor was calculated.

Sensitivity analyses were conducted, and all the models were used after (1) excluding early cases of incident hypertension (cases reported within the first 2 years of follow-up), (2) excluding late cases of hypertension (cases reported after $\geq 8$ years of follow-up), (3) excluding participants who reported incident chronic diseases during the follow-up period, (4) excluding participants who were under the 5th percentile and over the 95th percentile of total energy intake, (5) excluding participants who did not answer nine 
or more items in the FFQ, and (6) including participants who reported systolic blood pressure $\geq 140$ and/or diastolic blood pressure $\geq 90$ at baseline. Finally, an additional analysis was conducted, adjusting for the calendar year of participant's inclusion in the cohort.

All $P$ values presented are two-tailed; $P<0.05$ was considered statistically significant. The analyses were carried out using Stata/SE version 12.0 (StataCorp).

\section{Results}

A total of 5059 men and 8620 women were included in the present study. The main baseline characteristics of the participants according to their frequency of fried food consumption are given in Table 1 . The mean age of the participants was 36.5 (SD 10.8) years and the mean BMI was
$23 \cdot 2(\mathrm{sD} 3 \cdot 3) \mathrm{kg} / \mathrm{m}^{2}$. Participants in the highest category of baseline fried food consumption ( $>4$ times/week) compared with those in the lowest category ( $\leq 2$ times/week) had a higher BMI, were less physically active, were more likely to have a history of hypercholesterolaemia, but less likely to have a family history of hypertension, had higher total energy and fat intake, lower protein and carbohydrate intake, higher $\mathrm{Na}, \mathrm{Mg}$ and caffeine intake, but lower fibre, fruit, vegetable and low-fat dairy product intake, and had higher means of baseline systolic blood pressure and diastolic blood pressure.

During the follow-up period (median of 6.3 years), we identified 1232 incident cases of hypertension. A higher frequency of fried food consumption was positively associated with a higher risk of developing hypertension. Participants consuming fried foods $>2-4$ and $>4$ times/week exhibited

Table 1. Baseline characteristics of the participants according to their frequency of fried food consumption (the SUN (Seguimiento Universidad de Navarra) cohort, 1999-2012)

(Mean values, standard deviations and percentages)

\begin{tabular}{|c|c|c|c|c|c|c|}
\hline & \multicolumn{6}{|c|}{ Baseline consumption of fried foods (no. of times/week) } \\
\hline & \multicolumn{2}{|c|}{$0-2$ times/week } & \multicolumn{2}{|c|}{$>2-4$ times/week } & \multicolumn{2}{|c|}{$>4$ times/week } \\
\hline & Mean & SD & Mean & SD & Mean & SD \\
\hline$n$ & \multicolumn{2}{|c|}{5689} & \multicolumn{2}{|c|}{4520} & \multicolumn{2}{|c|}{3470} \\
\hline Age (years) & $36 \cdot 1$ & $10 \cdot 5$ & $36 \cdot 9$ & $10 \cdot 9$ & $36 \cdot 6$ & $11 \cdot 1$ \\
\hline Men (\%) & \multicolumn{2}{|c|}{$28 \cdot 7$} & \multicolumn{2}{|c|}{$39 \cdot 1$} & \multicolumn{2}{|c|}{47.8} \\
\hline BMI $\left(\mathrm{kg} / \mathrm{m}^{2}\right)$ & $22 \cdot 9$ & $3 \cdot 2$ & $23 \cdot 3$ & $3 \cdot 3$ & $23 \cdot 4$ & $3 \cdot 3$ \\
\hline \multicolumn{7}{|l|}{ Baseline BP (mmHg) } \\
\hline Systolic & $112 \cdot 3$ & $10 \cdot 8$ & 113.5 & $11 \cdot 2$ & 114.5 & $11 \cdot 0$ \\
\hline Diastolic & $68 \cdot 3$ & $8 \cdot 1$ & $69 \cdot 1$ & 8.2 & 69.6 & $8 \cdot 2$ \\
\hline Physical activity (MET/week) & $22 \cdot 2$ & 23.7 & $21 \cdot 2$ & $22 \cdot 0$ & $20 \cdot 2$ & $21 \cdot 3$ \\
\hline Hypercholesterolaemia (\%) & \multicolumn{2}{|c|}{$14 \cdot 3$} & \multicolumn{2}{|c|}{$12 \cdot 8$} & \multicolumn{2}{|c|}{14.6} \\
\hline Family history of hypertension (\%) & \multicolumn{2}{|c|}{$41 \cdot 0$} & \multicolumn{2}{|c|}{$41 \cdot 0$} & \multicolumn{2}{|c|}{38.0} \\
\hline \multicolumn{7}{|l|}{ Smoking status $(\%)$} \\
\hline Current & \multirow{2}{*}{\multicolumn{2}{|c|}{$\begin{array}{l}21.9 \\
28.5\end{array}$}} & \multirow{2}{*}{\multicolumn{2}{|c|}{$\begin{array}{l}23 \cdot 1 \\
25 \cdot 6\end{array}$}} & \multicolumn{2}{|c|}{$23 \cdot 0$} \\
\hline Former & & & & & \multicolumn{2}{|c|}{$26 \cdot 2$} \\
\hline TV watching (h/d) & 1.0 & 0.8 & 1.0 & $0 \cdot 8$ & 1.0 & 0.8 \\
\hline \multicolumn{7}{|l|}{ Total energy intake } \\
\hline $\mathrm{kJ} / \mathrm{d}$ & 9209 & 2456 & 10117 & $2464 \cdot 4$ & 10736 & $2489 \cdot 5$ \\
\hline $\mathrm{kcal} / \mathrm{d}$ & 2201 & 587 & 2418 & 589 & 2566 & 595 \\
\hline Macronutrients (\% energy) & & & & & & \\
\hline Carbohydrate intake & 44 & 8 & 44 & 7 & 43 & 7 \\
\hline Protein intake & 19 & 3 & 18 & 3 & 17 & 3 \\
\hline Fat intake & 36 & 7 & 37 & 7 & 38 & 6 \\
\hline SFA & 12 & 3 & 13 & 3 & 13 & 3 \\
\hline MUFA & 15 & 4 & 16 & 4 & 16 & 3 \\
\hline PUFA & 5 & 1 & 5 & 1 & 6 & 2 \\
\hline Alcohol intake & 2 & 3 & 2 & 3 & 2 & 3 \\
\hline Intake of & & & & & & \\
\hline $\mathrm{Na}(\mathrm{g} / \mathrm{d})$ & $3 \cdot 0$ & $2 \cdot 1$ & 3.5 & $2 \cdot 2$ & $3 \cdot 7$ & $2 \cdot 3$ \\
\hline$K(g / d)$ & 4.7 & 1.7 & 4.7 & 1.5 & 4.7 & 1.4 \\
\hline $\mathrm{Mg}(\mathrm{mg} / \mathrm{d})$ & $410 \cdot 3$ & $128 \cdot 8$ & $415 \cdot 8$ & $118 \cdot 7$ & $418 \cdot 1$ & $113 \cdot 5$ \\
\hline Fibre $(g / d)$ & $28 \cdot 2$ & $13 \cdot 1$ & $26 \cdot 8$ & 11.4 & $25 \cdot 9$ & $10 \cdot 6$ \\
\hline Caffeine (mg/d) & $42 \cdot 6$ & $39 \cdot 3$ & $44 \cdot 1$ & $39 \cdot 1$ & $46 \cdot 3$ & $40 \cdot 5$ \\
\hline Fruits $(\mathrm{g} / \mathrm{d})$ & 367.4 & $315 \cdot 7$ & 334.4 & 299.0 & 299.4 & $245 \cdot 3$ \\
\hline Vegetables $(g / d)$ & $573 \cdot 2$ & 371.0 & $495 \cdot 2$ & 294.8 & $467 \cdot 8$ & $299 \cdot 4$ \\
\hline Low-fat dairy products $(\mathrm{g} / \mathrm{d})$ & $258 \cdot 2$ & 254.9 & $213 \cdot 2$ & $242 \cdot 4$ & $181 \cdot 0$ & $230 \cdot 7$ \\
\hline High-fat dairy products (g/d) & $173 \cdot 7$ & $187 \cdot 7$ & $214 \cdot 7$ & $197 \cdot 4$ & $238 \cdot 3$ & $210 \cdot 9$ \\
\hline Sweets $(g / d)$ & $42 \cdot 0$ & 34.1 & $51 \cdot 2$ & 35.4 & $57 \cdot 2$ & 37.4 \\
\hline Soft drinks (ml/d) & $34 \cdot 1$ & 70.5 & $43 \cdot 1$ & $80 \cdot 8$ & $54 \cdot 1$ & $99 \cdot 0$ \\
\hline Fast foods $(\mathrm{g} / \mathrm{d})$ & $19 \cdot 8$ & $19 \cdot 3$ & 23.0 & $20 \cdot 8$ & $24 \cdot 2$ & $21 \cdot 3$ \\
\hline
\end{tabular}

BP, blood pressure; MET, metabolic equivalents; TV, television. 
Table 2. Cox proportional hazard ratios (HR) of incident hypertension according to the baseline frequency of fried food consumption in 13679 participants of the SUN (Seguimiento Universidad de Navarra) project (1999-2012) (Hazard ratios and $95 \%$ confidence intervals)

\begin{tabular}{|c|c|c|c|c|c|c|}
\hline & \multicolumn{6}{|c|}{ Baseline consumption of fried foods (no. of times/week) } \\
\hline & \multirow[b]{2}{*}{$0-2$ times/week } & \multicolumn{2}{|c|}{$>2-4$ times/week } & \multicolumn{2}{|c|}{$>4$ times/week } & \multirow[b]{2}{*}{$P$ for trend } \\
\hline & & $\mathrm{HR}$ & $95 \% \mathrm{Cl}$ & HR & $95 \% \mathrm{Cl}$ & \\
\hline$n$ & 419 & & 141 & & 72 & \\
\hline Person-years & 39102 & & 403 & & 581 & \\
\hline Crude & 1.00 (ref.) & 1.31 & $1 \cdot 14,1.49$ & 1.38 & $1.20,1.59$ & $<0.001$ \\
\hline Age- and sex-adjusted model & 1.00 (ref.) & $1 \cdot 19$ & $1.04,1.37$ & $1 \cdot 19$ & $1.04,1.38$ & 0.017 \\
\hline Multivariable-adjusted model ${ }^{*}$ & 1.00 (ref.) & $1 \cdot 20$ & $1.04,1.37$ & $1 \cdot 18$ & $1.01,1.38$ & 0.042 \\
\hline Multivariable-adjusted model $\dagger$ & 1.00 (ref.) & $1 \cdot 18$ & $1.03,1.36$ & 1.21 & $1.04,1.41$ & 0.009 \\
\hline
\end{tabular}

ref., Reference.

${ }^{*}$ Adjusted for sex, age, family history of hypertension, self-reported hypercholesterolaemia, physical activity, smoking status, total energy intake, alcohol intake, energy-adjusted $\mathrm{Na}, \mathrm{K}$, caffeine, fibre, olive oil, fruit, vegetable, low-fat and high-fat dairy product, sugar-sweetened beverage, fast food, and sweet consumption, and time spent watching television.

† Additionally adjusted for baseline BMI.

a significantly greater risk of developing hypertension compared with those consuming fried foods $0-2$ times/week (hazard ratio $(\mathrm{HR})=1 \cdot 18,95 \% \mathrm{CI} 1 \cdot 03,1.36$ and $\mathrm{HR}=1 \cdot 21$, 95\% CI 1.04, 1.41, respectively; $P$ for trend=0.009), after adjusting for potential confounders (Table 2). When analysing total fried food consumption $(\mathrm{g} / \mathrm{d})$ in tertiles, we observed a HR of $1.04(95 \%$ CI $0.90,1.20)$ for the second tertile and a HR of 1.18 (95\% CI $1.00,1.38)$ for the third tertile $v$. the first tertile of consumption in the fully adjusted models (data not shown). The Pearson correlation coefficient between the frequency of fried food consumption and total fried food consumption in grams was $r 0.912(P<0 \cdot 001)$. When analysing the four categories of consumption, we also observed a greater risk in the highest category ( $\geq 7$ times/week) in comparison with the lowest category $(\mathrm{HR}=1 \cdot 20,95 \% \mathrm{CI} 1 \cdot 00,1 \cdot 45 ; P$ for trend $=0 \cdot 017)$ (data not shown).

As we thought that the intake of this type of food might change over time and/or over the study period (i.e. early $v$. late entry into the dynamic cohort), we conducted an additional analysis adjusting for the calendar year of participant's inclusion in the cohort. We observed that the HR were attenuated, but remained statistically significant at 1.15 (95\% CI 1.00, 1.31) and 1.16 (95\% CI 1.01, 1.34) for those consuming fried foods $>2-4$ times/week and $>4 v .<2$ times/week, respectively.

When analysing the frequency of fried food consumption at home and away from home, we obtained very similar results for both groups. We observed a HR of 1.16 for those consuming fried foods at home $>2$ times/week (95\% CI 1.03, 1.32) and a HR of 1.15 for those consuming fried foods away from home (95\% CI 0.97, 1.38) (Table 3).

The interactions between fried food consumption and age, sex and type of oil used for frying were not statistically significant ( $P$ for interaction $=0.711,0.567$ and 0.870 , respectively).

We conducted multiple sensitivity analyses to account for the potential uncertainties of our assumptions regarding the induction period and also for possible sources of bias including measurement errors. In all these sensitivity analyses, the results were not meaningfully altered (Table 4).

When testing for multicollinearity, we found variance inflation factor values to be satisfactory.

\section{Discussion}

Higher baseline consumption of fried foods was found to be significantly associated with a higher risk of incident hypertension in the Mediterranean cohort studied.

A previous cross-sectional study carried out in Spain including 538 participants ${ }^{(13)}$ has evaluated the association between the consumption of fried foods and the prevalence

Table 3. Cox proportional hazard ratios (HR) of incident hypertension according to the baseline frequency of fried food consumption, stratified according to fried food consumption at home or away from home (Hazard ratios and $95 \%$ confidence intervals)

\begin{tabular}{|c|c|c|c|}
\hline & \multicolumn{3}{|c|}{$\begin{array}{l}\text { Baseline consumption of fried } \\
\text { foods (no. of times/week) }\end{array}$} \\
\hline & \multirow{2}{*}{$\begin{array}{l}0-2 \text { times/ } \\
\text { week }\end{array}$} & \multicolumn{2}{|c|}{$>2$ times/week } \\
\hline & & $\mathrm{HR}$ & $95 \% \mathrm{Cl}$ \\
\hline \multicolumn{4}{|l|}{ Fried food consumption at home* } \\
\hline$n$ & 449 & \multicolumn{2}{|r|}{777} \\
\hline Person-year & 41323 & \multicolumn{2}{|r|}{56382} \\
\hline Crude & 1.00 (ref.) & 1.32 & $1.18,1.48$ \\
\hline Age- and sex-adjusted model & 1.00 (ref.) & $1 \cdot 16$ & $1.04,1.31$ \\
\hline Multivariable-adjusted model $\dagger$ & 1.00 (ref.) & $1 \cdot 16$ & $1.02,1.31$ \\
\hline Multivariable-adjusted model $\ddagger$ & 1.00 (ref.) & $1 \cdot 16$ & $1.03,1.32$ \\
\hline \multicolumn{4}{|c|}{ Fried food consumption away from home§ } \\
\hline$n$ & 1041 & \multirow{2}{*}{\multicolumn{2}{|c|}{9791}} \\
\hline Person-year & 86654 & & \\
\hline Crude & 1.00 (ref.) & 1.28 & $1.08,1.52$ \\
\hline Age- and sex-adjusted model & 1.00 (ref.) & $1 \cdot 22$ & $1.03,1.46$ \\
\hline Multivariable-adjusted model $\dagger$ & 1.00 (ref.) & $1 \cdot 18$ & $0.99,1.41$ \\
\hline Multivariable-adjusted model $\ddagger$ & 1.00 (ref.) & $1 \cdot 16$ & $0.97,1.38$ \\
\hline
\end{tabular}

ref., Reference.

* We included 13629 participants in this analysis (fifty participants excluded due to missing information for this variable) and we identified 1226 incident cases of hypertension.

† Adjusted for sex, age, family history of hypertension, self-reported hypercholesterolaemia, physical activity, smoking status, total energy intake, alcohol intake, energy-adjusted $\mathrm{Na}, \mathrm{K}$, caffeine, fibre, olive oil, fruit, vegetable, low-fat and highfat dairy product, sugar-sweetened beverage, fast food, and sweet consumption, and time spent watching television.

$\ddagger$ Additionally adjusted for baseline BMI.

$\S$ We included 13453 participants in this analysis (226 participants were excluded due to missing information for this variable) and we identified 1190 incident cases of hypertension. 
Table 4. Cox model multivariate-adjusted hazard ratios (HR) of hypertension associated with the highest category ( $>4$ times/week) of fried food consumption in sensitivity analyses, taking the group with the lowest frequency of fried food consumption as the reference category ( $<2$ times/week) (Hazard ratios and $95 \%$ confidence intervals)

\begin{tabular}{|c|c|c|c|c|c|c|}
\hline & $n$ & No. of HTA cases & $\%$ & $\mathrm{HR}^{*}$ & $95 \% \mathrm{Cl}$ & $P$ for trend \\
\hline Overall & 13679 & 1232 & $9 \cdot 0$ & $1 \cdot 22$ & $1.04,1.42$ & 0.015 \\
\hline \multicolumn{7}{|l|}{ Sensitivity analyses } \\
\hline $\begin{array}{l}\text { Considering early cases of hypertension } \\
\text { as non-cases }\end{array}$ & 13679 & 1002 & $7 \cdot 3$ & $1 \cdot 25$ & $1.06,1.48$ & 0.011 \\
\hline $\begin{array}{l}\text { Considering late cases of hypertension } \\
\text { as non-cases }\end{array}$ & 13679 & 962 & $7 \cdot 0$ & $1 \cdot 25$ & $1.05,1.49$ & 0.011 \\
\hline $\begin{array}{l}\text { Excluding all incident cases of } \\
\text { chronic disease from the analyses }\end{array}$ & 13111 & 1120 & 8.5 & 1.14 & $0.97,1.34$ & 0.181 \\
\hline $\begin{array}{l}\text { Setting different energy limits: } \\
\text { percentiles } 5-95\end{array}$ & 13698 & 1192 & $8 \cdot 7$ & $1 \cdot 22$ & $1.05,1.43$ & 0.020 \\
\hline $\begin{array}{l}\text { Excluding participants who left more } \\
\text { than nine items blank in } \\
\text { the FFQ }\end{array}$ & 11246 & 928 & $8 \cdot 2$ & $1 \cdot 28$ & $1.07,1.52$ & 0.043 \\
\hline $\begin{array}{l}\text { Including those who reported SBP } \\
\geq 140 \mathrm{mmHg} \text { and/or DBP } \geq 90 \mathrm{mmHg} \text { at baseline }\end{array}$ & 14033 & 1379 & $9 \cdot 8$ & $1 \cdot 19$ & $1.03,1.38$ & 0.026 \\
\hline
\end{tabular}

SBP, systolic blood pressure; DBP, diastolic blood pressure.

*Adjusted for sex, age, baseline BMI, family history of hypertension, self-reported hypercholesterolaemia, total energy intake, smoking status, alcohol intake, energy-adjusted $\mathrm{Na}, \mathrm{K}$, caffeine, fibre, olive oil, fruit, vegetable, low-fat and high-fat dairy product consumption, sugar-sweetened beverage, fast food, and sweet consumption, and time spent watching television.

of hypertension and found the risk of hypertension to be directly associated with the consumption of fried foods, especially on reusing vegetable oils such as sunflower oil (13). Some studies have evaluated the association between the consumption of fried foods and the risk of CVD with inconsistent results ${ }^{(14-17)}$. The Spanish EPIC cohort study, conducted in 40757 adults aged 29-69 years, free of CHD at baseline, found that the consumption of fried foods was not associated with the risk of CHD with a HR of 1.08 (95\% CI 0.82, 1.43) for the fourth quartile of consumption in comparison with the first quartile ${ }^{(16)}$. Similarly, a case-control study carried out in Costa Rica found no association between the consumption of fried foods and the risk of myocardial infarction ${ }^{(17)}$. Conversely, the large INTERHEART case-control study observed a positive association between the consumption of fried foods and the risk of acute myocardial infarction ${ }^{(14)}$. The Cardiovascular Heart Study found fried fish consumption to exhibit a nonsignificant trend towards a higher risk ${ }^{(15)}$.

The frying process involves many factors, some dependent on the process itself and others on the food and type of fat used $^{(7)}$. For example, during the frying process, the amount of trans-fatty acids in foods increases. The amount of transfatty acids generated depends on several aspects such as the frying technique (deep frying or pan frying), the type of oil and food used, and the degree of degradation of the oil ${ }^{(6)}$. In one large American cohort that included 28100 women aged $\geq 39$ years and free of CVD and cancer at baseline, Wang et $a l{ }^{(30)}$ found a higher intake of trans-fatty acids to be associated with an increased risk of hypertension. Therefore, this mechanism may account for the association found in the present study. Another plausible mechanism involved in the association between fried food consumption and hypertension risk might be explained by the weight gained during the follow-up period due to fried food consumption. A previous study carried out in our cohort has shown a significant positive association between high frequency of fried food consumption and the risk of overweight/obesity ${ }^{(8)}$. The association between hypertension and obesity is widely known ${ }^{(31)}$. In this context, we conducted a secondary analysis, additionally adjusting for incident obesity (yes/no). We found the risk estimate to be attenuated and the association between fried food consumption $(>4 v$. $\leq 2$ times/week) and hypertension risk to be non-significant (HR $=1 \cdot 15,95 \%$ CI 0.97, 1.36). Similar results were obtained when we adjusted for yearly body weight change ( $\mathrm{HR}=1 \cdot 14,95 \% \mathrm{CI}, 0 \cdot 96,1 \cdot 46)$. Therefore, incident obesity and yearly body weight change might be mediators in the causal chain, and this is likely to act as one of the potential mechanisms that might explain the association between the consumption of fried foods and the incidence of hypertension.

A potential limitation of the present study is the self-reported outcome. However, a self-reported diagnosis of hypertension has been validated previously in our cohort and a fairly adequate validity for the self-reported diagnosis of hypertension found ${ }^{(27,28)}$

Another limitation could be that the participants might have under-reported their fried food consumption because they perceived it as unhealthy (social desirability bias); however, this misclassification is expected to be non-differential and therefore could more likely bias the results of the present study to the null value ${ }^{(32)}$

The present study also has important strengths, including its prospective design, which avoids the possibility of reverse causation bias. Other strengths are the use of a validated $\mathrm{FFQ}^{(22)}$, the large sample size and the long follow-up period.

Inclusion of only university graduates might be a potential limitation of the present study, because it is not a representative sample. This might have affected the generalisability of our findings; therefore, we have to be cautious while extrapolating the results of the present study to the general population. Nevertheless, it could also have actually enhanced the internal validity of the present study because the high level of education 
and homogeneity of the cohort reduced the potential confounding related to socio-economic status. However, the generalisability of our findings, to some other important cohort, must be based on biological mechanisms and not on the representativeness of the study sample in the statistical sense of the term. In addition, the high educational level of the study participants helped us obtain better-quality information and increased the internal validity of the study.

In conclusion, more frequent consumption of fried foods at baseline was found to be associated with a higher risk of developing hypertension during the follow-up period in a Mediterranean cohort of university graduates.

\section{Supplementary material}

To view supplementary material for this article, please visit http://dx.doi.org/10.1017/S00071145140001755

\section{Acknowledgements}

The authors thank all the participants of the SUN project for their continued cooperation and participation. They also thank all the members of the SUN project for administrative, technical and material support.

The SUN study has received funding from the Spanish Government (grant nos. PI01/0619, PI030678, PI040233, PI042241, PI050976, PI070240, PI070312, PI081943, PI080819, PI1002658, PI1002293, RD06/0045, G03/140 and 87/2010), the Navarra Regional Government (36/2001, 43/2002, 41/2005, 36/2008, $45 / 2011$ ), and the University of Navarra. A. G. is supported by a FPU fellowship of the 'Ministerio de Educación, Cultura y Deporte', from the Spanish Government.

The authors' contributions are as follows: M. A. M.-G. helped design the study and collected the data; M. B.-R. and C. S.-O. analysed the data and drafted the manuscript; A. G., I. Z., F. J. B.-G. edited and critically reviewed the manuscript. None of the authors has any conflicts of interest to declare.

\section{References}

1. Lloyd Jones D, Adams R, Brown T, et al. (2010) Heart disease and stroke statistics -2010 update: a report from the American Heart Association. Circulation 121, e46-e215.

2. World Health Organization (2009) Global Health Risk: Mortality and Burden of Disease Attributable to Selected Major Risk. Geneva: World Health Organization.

3. Lim S, Vos T, Flaxman A, et al. (2012) A comparative risk assessment of burden of disease and injury attributable to 67 risk factors and risk factor clusters in 21 regions, 19902010: a systematic analysis for the Global Burden of Disease Study 2010. Lancet 380, 2224-2260.

4. World Health Organization (2008) Causes of Death 2008 [Online Database]. Geneva: World Health Organization. http://www.who.int/healthinfo/global_burden_disease/cod_ 2008_sources_methods.pdf

5. Ibrahim MM \& Damasceno A (2012) Hypertension in developing countries. Lancet 380, 611-619.

6. Boskou G, Salta F, Chiou A, et al. (2006) Content of trans, trans-2,4-decadienal in deep-fried and pan-fried potatoes. Eur J Lipd Sci Technol 108, 109-115.
7. Saguy IS \& Pinthus IJ (1995) Oil uptake during deep-fat frying: factors and mechanism. Food Technol 49, 142-145.

8. Sayon-Orea C, Bes-Rastrollo M, Basterra-Gortari FJ, et al. (2013) Consumption of fried foods and weight gain in a Mediterranean cohort: the SUN project. Nutr Metab Cardiovasc Dis 23, 144-150.

9. Soriguer F, Almaraz MC, García-Almeida JM, et al. (2010) Intake and home use of olive oil or mixed oils in relation to healthy lifestyles in a Mediterranean population. Findings from the prospective Pizarra study. Br J Nutr 103, $114-122$.

10. Jahren AH \& Schubert BA (2010) Corn content of French fry oil from national chain vs. small business restaurants. Proc Natl Acad Sci U S A 107, 2099-2101.

11. Guallar-Castillón P, Rodríguez-Artalejo F, Fornés $\mathrm{N}$, et al. (2007) Intake of fried foods is associated with obesity in the cohort of Spanish adults from the European Prospective Investigation into Cancer and Nutrition. Am J Clin Nutr 86, $198-205$.

12. Gerber RT, Holemans K, O'Brien Coker I, et al. (1999) Cholesterol-independent endothelial dysfunction in virgin and pregnant rats fed a diet high in saturated fat. $J$ Physiol 517, $607-616$.

13. Soriguer F, Rojo-Martnez G, Dobarganes MC, et al. (2003) Hypertension is related to the degradation of dietary frying oils. Am J Clin Nutr 78, 1092-1097.

14. Iqbal R, Anand S, Ounpuu S, et al. (2008) Dietary patterns and the risk of acute myocardial infarction in 52 countries: results of the INTERHEART study. Circulation 118, 1929-1937.

15. Mozaffarian D, Lemaitre R, Kuller L, et al. (2003) Cardiac benefits of fish consumption may depend on the type of fish meal consumed: the Cardiovascular Health Study. Circulation 107, 1372-1377.

16. Guallar-Castillon P, Rodriguez-Artalejo F, Lopez Garcia E, et al. (2012) Consumption of fried foods and risk of coronary heart disease: Spanish cohort of the European Prospective Investigation into Cancer and Nutrition study. BMJ $\mathbf{3 4 4}$, e363.

17. Kabagambe EK, Baylin A, Siles X, et al. (2003) Individual saturated fatty acids and nonfatal acute myocardial infarction in Costa Rica. Eur J Clin Nutr 57, 1447-1457.

18. Gu D, Wildman RP, Wu X, et al. (2007) Incidence and predictors of hypertension over 8 years among Chinese men and women. J Hypertens 25, 517-523.

19. Kisioglu AN, Aslan B, Ozturk M, et al. (2004) Improving control of high blood pressure among middle-aged Turkish women of low socio-economic status through public health training. Croat Med J 45, 477-482.

20. Segui-Gomez M, de la Fuente C, Vazquez Zenaida, et al. (2006) Cohort profile: the 'Seguimiento Universidad de Navarra' (SUN) study. Int J Epidemiol 35, 1417-1422.

21. Willett WC \& Stampfer M (1998) Implications of total energy intake for epidemiologic analyses. In Nutritional Epidemiology, 2nd ed., pp. 273-301 [WC Willett, editor]. New York: Oxford University Press.

22. Martín-Moreno JM, Boyle P, Gorgojo L, et al. (1993) Development and validation of a food frequency questionnaire in Spain. Int J Epidemiol 22, 512-519.

23. Moreiras O, Carbajal A, Cabrera L, et al. (2007) Las tablas (Tables). In Tabla de composicion de alimentos (Food Composition Table), 11th ed., pp. 37-46 [O Moreiras, A Carbajal, L Cabrera and C Cuadrado, editors]. Madrid: Pirámide.

24. Bes-Rastrollo M, Pérez-Valdivieso JR, Sanchez-Villegas A, et al. (2005) Validación del peso e índice de masa corporal 
auto-declarados de los participantes de una cohorte de graduados universitarios (Validation of self-reported weight and body mass index in a cohort of university graduates). Rev Esp Obes 3, 183-189.

25. Martinez-Gonzalez MA, López-Fontana C, Varo JJ, et al. (2005) Validation of the Spanish version of the physical activity questionnaire used in the Nurses' Health Study and the Health Professionals' Follow-up Study. Public Health Nutr 8, 920-927.

26. Chobanian A, Bakris G, Black H, et al. (2003) Seventh report of the Joint National Committee on Prevention, Detection, Evaluation, and Treatment of High Blood Pressure. Hypertension 42, 1206-1252.

27. Alonso A, Beunza J, Delgado-Rodriguez M, et al. (2005) Validation of self-reported diagnosis of hypertension in a cohort of university graduates in Spain. BMC Public Health 5, 94.
28. Fernandez-Montero A, Beunza J, Bes Rastrollo $\mathrm{M}$, et al. (2011) [Validity of self-reported metabolic syndrome components in a cohort study]. Gac Sanit 25, 303-307.

29. Hernán MA, Hernández-Díaz S, Werler MM, et al. (2002) Causal knowledge as a prerequisite for confounding evaluation: an application to birth defects epidemiology. $A m \mathrm{~J}$ Epidemiol 155, 176-184.

30. Wang L, Manson J, Forman J, et al. (2010) Dietary fatty acids and the risk of hypertension in middle-aged and older women. Hypertension 56, 598-604.

31. Landsberg L, Aronne L, Beilin L, et al. (2013) Obesity-related hypertension: pathogenesis, cardiovascular risk, and treatment: a position paper of the Obesity Society and the American Society of Hypertension. J Clin Hypertens 15, 14-33.

32. Rothman KJ, Greenland S \& Lash TL (2008) Modern Epidemiology, 3rd ed. Philadelphia, PA: Lippincott Williams and Wilkins. 